\title{
Predicting cardiac events using ventricular dyssynchrony in patients who received implantable cardioverter defibrillators: Are more treatment options required?
}

\author{
Keiichiro Yoshinaga, MD, PhD, FACC \\ ${ }^{a}$ Molecular Imaging Research Center, National Institute of Radiological Sciences, Chiba, Japan
}

Received Nov 3, 2015; accepted Nov 3, 2015

doi:10.1007/s12350-015-0326-x

\section{See related article, pp. 122-129}

The survival rate associated with heart failure (HF) has been significantly improved in recent years due to improved preventive approaches and device treatments to improve cardiac function and prevent sudden cardiac death. ${ }^{1}$ Implantable devices used in cardiac resynchronization therapy (CRT) and implantable cardioverter defibrillators (ICDs) contribute to improving outcomes for HF patients. The use of ICD implantation has been stable over the years and overall the ICD has resulted in reduced mortality. ${ }^{2}$ However, it is true that limited numbers of patients have benefited from ICD implantation. ${ }^{3}$ A meta-analysis showed that some sub-study populations had no benefits from ICD implantation. ${ }^{4}$ At present, ICD implantation may be the best therapeutic option for preventing sudden cardiac death in patients with left ventricular ejection fraction (LVEF) of $<35 \%$. If predictors for ICD effectiveness or non-response can be identified, other treatment approaches may be available to the non-responder. In addition, with implantable devices, infective endocarditis can be a cause for major concern, and therefore unnecessary or ineffective implantation should be avoided. ${ }^{5,6}$ Diagnostic approaches have therefore been developed in order to select suitable patients for ICD treatments. Nuclear cardiology imaging has contributed to predicting an

Reprint requests: Keiichiro Yoshinaga, MD, PhD, FACC, Molecular Imaging Research Center, National Institute of Radiological Sciences, 4-9-1 Anagawa, Inage-Ku, Chiba 263-8555, Japan; kyoshi@nirs.go.jp

J Nucl Cardiol 2017:24;130-3.

$1071-3581 / \$ 34.00$

Copyright (C) 2015 American Society of Nuclear Cardiology. individual's response to ICD treatments (Table 1)..$^{3,7,8}$ Recently, Fallavollita et al reported that sympathetic denervation predicts cause-specific mortality from sudden cardiac death independently of LVEF in patients with coronary artery disease (CAD) and LV dysfunction. ${ }^{8}$ Cardiac sympathetic nervous function was evaluated using ${ }^{11} \mathrm{C}$-hydroxyephedrine (HED), which reflects presynaptic sympathetic nervous function. 9,10 This approach may be able to detect patients who are at higher risk for arrhythmia-induced sudden cardiac death regardless of LVEF. ACC/AHA guidelines for the appropriate use of implantable defibrillators address the importance of detecting LV dyssynchrony. ${ }^{11} \mathrm{LV}$ dyssynchrony was previously evaluated mainly by echocardiography. ${ }^{12}$

Using gated single-photon emission computed tomography (SPECT), LV dyssynchrony can be easily and reliably estimated. ${ }^{13,14}$ Dyssynchrony is defined as "ventricular electromechanical delay". Prolongation of the QRS complex is seen in approximately one-third of patients with advanced $\mathrm{HF}$, and this prolongation may be associated with varying degrees of ventricular electromechanical delay. Among several dyssynchrony parameters, the phase standard deviations (SD) and bandwidth have been most useful for differentiating between patients with and without dyssynchrony. ${ }^{15}$ Araujo et al evaluated the usefulness of dyssynchrony parameters in patients who had ICDs. At 1 year, patients with events had higher phase SD and higher histogram bandwidth than did patients without events. ${ }^{7}$ This previous study raised the research question of whether dyssynchrony can be a predictor of outcomes in patients with ICD implantation.

In this issue of Journal of Nuclear Cardiology, Zafrir et al evaluated the predictive value of LV mechanical dyssynchrony for major cardiac events (MACE) in patients who had ICD implantation. ${ }^{16}$ At a 
Table 1. PET and SPECT for patients with implantable ICDs

\begin{tabular}{|c|c|c|}
\hline Tests & Pathophysiology & Indication \\
\hline${ }^{99}{ }^{\mathrm{T}} \mathrm{Tc}$ RBC RNA & LV systolic function measurements & ICDs indication \\
\hline${ }^{11} \mathrm{C}$-hydroxyephedrine & Sympathetic nervous function & Predicting sudden cardiac death \\
\hline${ }^{99 \mathrm{~m}}$ Tc-sestamibi & LV dyssynchrony & Possibly predicting major cardiac events \\
\hline${ }^{18} \mathrm{~F}$-fluorodeoxyglucose & Inflammation & Mechanical lead infection \\
\hline
\end{tabular}

ICDs implantable cardioverter defibrillators, $L V$ left ventricle, $R B C$ red blood cell, $R N A$ radionuclide angiography

mean point of 23.7 months, the authors followed up the study population minus those patients who were lost to follow-up. All mortality causes and other major cardiac events were examined and all patients were closely observed by a heart failure clinic or arrhythmia clinic. Such strong follow-up surely strengthens the quality of evidence, something that is often a challenge with prognostic studies. ${ }^{17}$ As mentioned before, some previous studies looked at the prognostic value of ventricular dyssynchrony, detected using gated SPECT myocardial perfusion imaging (MPI), in heart failure. ${ }^{18}$ However, the prognostic value of ventricular dyssynchrony in specific population such as patients with ICD implantation has not been fully studied. The most important finding of the present study by Zafrir et al that the authors evaluated was the prognostic value of LV ventricular dyssynchrony in patients with ICD implantation. When the phase cut-off value of $60^{\circ}$ was applied, patients who had cardiac death and progression of congestive heart failure (CHF) were able to be differentiated from patients without such cardiac events. However, this cut-off value could not be used to differentiate between patients with ventricular tachycardia (VT)/ventricular fibrillation (VF) events and patients without VT/VF events. This is the difference between the current study and a recent study by Aljaroudi et al. ${ }^{7,16}$ These data may imply that abnormal LV mechanical dyssynchrony was associated with developing cardiac dysfunction rather than with increasing life-threatening arrhythmia in this study population. In addition, a Kaplan-Meier survival curve clearly separated the cardiac death group and the survival group based on LV mechanical dyssynchrony cut-off value. This cut-off value could also be used to effectively differentiate between the deteriorating $\mathrm{HF}$ and stable HF groups in the ICD population. There was no difference in LV mechanical dyssynchrony between the VT/VF event group and the group without VT/VF events. Based on these current data, LV mechanical dyssynchrony may be an important predictor of developing LV dysfunction without wide QRS complex in those patients who required ICD implantation. The developing LV dysfunction seems to be associated with subsequent cardiac death. However, the authors did not provide detailed causes of death in this issue of Journal of Nuclear Cardiology. Thus, these postulations are speculation and the authors are expected to provide these important data as part of their future work.

Another important finding of the current study should be the independent predictors of cardiac death in the ICD implant population. The independent predictors include wide QRS complex and LV mechanical dyssynchrony. Current ACC/AHA guidelines for the appropriate use of ICD implantation stipulate the presence of reduced LVEF. ${ }^{11}$ There are many controversial opinions regarding this ICD indication, and many cardiologists are seeking better or alternative indications for ICD implantation. The current data showed that reduced LVEF was not significant for predicting cardiac death using either univariate analysis or multivariate analysis. Although the numbers of the study population were limited, this finding strongly suggests that other indications for ICD implantation should be sought.

Despite the important findings by Zafrir et al, several critical issues remain. First, the authors applied a phase SD of $60^{\circ}$ as a cut-off value based on their experiences. However, this cut-off value does not seem to have been validated. Although the results of the current study showed that this cut-off value could be used to distinguish between patients with cardiac events and those with non-cardiac events, this cut-off value needs to be validated by other standard measurements. Second, the authors included coronary artery disease and non-ischemic heart failure in this study. Some of the patients had stress and rest myocardial perfusion imaging and the remaining patients had only rest imaging. Myocardial ischemia and viable myocardium not treated by revascularization are also associated with significant deterioration of the LV function over time and increased cardiovascular events. ${ }^{19}$ In fact, Buxton et al reported 
that the sudden cardiac death ratio increased in CAD patients and that the mortality did not depend on the LVEF in CAD. ${ }^{20}$ In the current study, some patients who had positive summed difference scores may have required revascularization. In this regard, if the authors truly aimed to show the prognostic value of $\mathrm{LV}$ dyssynchrony in patients with ICD implantation, patients shown to have myocardial ischemia should have been excluded. Third, there seem to be many differences in baseline variables between the cardiac event group and non-event group. However, the authors performed only unadjusted Kaplan-Meier survival analysis. Although the size of the study population was sufficient considering the specific study population, the sample size and/or the number of major cardiac events may have been small for adjusted survival analysis. That being the case, this study needs further analysis to confirm the current results using appropriate adjusted survival analysis. Finally, the authors noted that patients who had greater dyssynchrony without left bundle branch block (LBBB) or wide QRS complex may require a CRT defibrillator (CRT-D) instead of an ICD. This question has just been raised in light of the current data. ACC/AHA guidelines for the appropriate use of ICDs recommend the application of dyssynchrony data in decision-making. ${ }^{11}$ To confirm their hypothesis, the authors may conduct follow-up rest ECG, LVEF, and look at whether the QRS complex became wider than $120 \mathrm{~mm}$ per second in patients who showed significant LV dyssynchrony at the baseline without wide QRS.

Based on the recent development of HF treatments, especially those involving implanted devices, the next step in HF device treatments should be to improve the effectiveness of these devices. In this regard, selecting suitable patients or developing appropriate indications for these devices should be considered to be further improvements in the quality of HF patient care. The findings of Zafrir et al thus represent a significant contribution towards establishing additional or alternative indications for ICD implantation in patients with HF. This study presents several ideas to our societies and will certainly lead to a number of related research projects on these important issues.

\section{Acknowledgments}

This manuscript has been reviewed by a North American English-language professional editor, Ms. Holly Beanlands. The authors also thank Ms. Holly Beanlands for critical reading of the manuscript.

\section{Disclosure}

The authors have indicated that they have no financial conflict of interest.

\section{References}

1. Owens AT, Jessup M. The year in heart failure. J Am Coll Cardiol 2012;60:359-68.

2. Borne RT, Peterson PN, Greenlee R, Heidenreich PA, Wang Y, Curtis JP, et al. Temporal trends in patient characteristics and outcomes among Medicare beneficiaries undergoing primary prevention implantable cardioverter-defibrillator placement in the United States, 2006-2010: Results from the National Cardiovascular Data Registry's Implantable Cardioverter-Defibrillator Registry. Circulation 2014;130:845-53.

3. Fisher JD, Buxton AE, Lee KL, Packer DL, Echt DS, Denes P, et al. Designation and distribution of events in the Multicenter UnSustained Tachycardia Trial (MUSTT). Am J Cardiol 2007;100:76-83.

4. Earley A, Persson R, Garlitski AC, Balk EM, Uhlig K. Effectiveness of implantable cardioverter defibrillators for primary prevention of sudden cardiac death in subgroups a systematic review. Ann Int Med 2014;160:111-21.

5. Leccisotti L, Perna F, Lago M, Leo M, Stefanelli A, Calcagni ML, et al. Cardiovascular implantable electronic device infection: Delayed vs standard FDG PET-CT imaging. J Nucl Cardiol 2014;21:622-32.

6. Sarrazin JF, Philippon F, Tessier M, Guimond J, Molin F, Champagne $\mathrm{J}$, et al. Usefulness of fluorine-18 positron emission tomography/computed tomography for identification of cardiovascular implantable electronic device infections. J Am Coll Cardiol 2012;59:1616-25.

7. Aljaroudi WA, Hage FG, Hermann D, Doppalapudi H, Venkataraman R, Heo J, et al. Relation of left-ventricular dyssynchrony by phase analysis of gated SPECT images and cardiovascular events in patients with implantable cardiac defibrillators. J Nucl Cardiol 2010;17:398-404.

8. Fallavollita JA, Heavey BM, Luisi AJ Jr, Michalek SM, Baldwa S, Mashtare TL Jr, et al. Regional myocardial sympathetic denervation predicts the risk of sudden cardiac arrest in ischemic cardiomyopathy. J Am Coll Cardiol 2014;63:141-9.

9. Thackeray JT, Bengel FM. Assessment of cardiac autonomic neuronal function using PET imaging. J Nucl Cardiol 2013;20:150-65.

10. Yoshinaga K, Tomiyama Y, Manabe O, Kasai K, Katoh C, Magota $\mathrm{K}$, et al. Prone-position acquisition of myocardial (123)Imetaiodobenzylguanidine (MIBG) SPECT reveals regional uptake similar to that found using (11)C-hydroxyephedrine PET/CT. Ann Nucl Med 2014;28:761-9.

11. Russo AM, Stainback RF, Bailey SR, Epstein AE, Heidenreich PA, Jessup M, et al. ACCF/HRS/AHA/ASE/HFSA/SCAI/SCCT/ SCMR 2013 appropriate use criteria for implantable cardioverterdefibrillators and cardiac resynchronization therapy: A report of the American College of Cardiology Foundation appropriate use criteria task force, Heart Rhythm Society, American Heart Association, American Society of Echocardiography, Heart Failure Society of America, Society for Cardiovascular Angiography and Interventions, Society of Cardiovascular Computed Tomography, and Society for Cardiovascular Magnetic Resonance. J Am Coll Cardiol 2013;61:1318-68.

12. Bax JJ, Abraham T, Barold SS, Breithardt OA, Fung JW, Garrigue $S$, et al. Cardiac resynchronization therapy: Part 2-issues during and after device implantation and unresolved questions. J Am Coll Cardiol 2005;46:2168-82.

13. Boogers MM, Van Kriekinge SD, Henneman MM, Ypenburg C, Van Bommel RJ, Boersma E, et al. Quantitative gated SPECTderived phase analysis on gated myocardial perfusion SPECT detects left ventricular dyssynchrony and predicts response to cardiac resynchronization therapy. J Nucl Med 2009;50:718-25. 
14. Tanaka T, Chikamori T, Hida S, Igarashi Y, Shiba C, Usui Y, et al. Diagnostic value of vasodilator-induced left ventricular dyssynchrony as assessed by phase analysis to detect multivessel coronary artery disease. Ann Nucl Cardiol 2015;1:6-17.

15. Chen J, Faber TL, Cooke CD, Garcia EV. Temporal resolution of multiharmonic phase analysis of ECG-gated myocardial perfusion SPECT studies. J Nucl Cardiol 2008;15:383-91.

16. Zafrir N, Bental T, Strasberg B, Solodky A, Mats I, Gutstin A et al. Yield of left ventricular dyssynchrony by gated SPECT MPI in patients with heart failure prior to implantable cardioverter defibrillator or cardiac resynchronization therapy with a defibrillator: Characteristics and prediction of cardiac outcomes. J Nucl Cardiol 2015. doi:10.1007/s12350-015-0310-5.

17. Yoshinaga K, Chow BJ, Williams K, Chen L, Dekemp RA, Gar$\operatorname{rard} \mathrm{L}$, et al. What is the prognostic value of myocardial perfusion imaging using rubidium-82 positron emission tomography? J Am Coll Cardiol 2006;48:1029-39.

18. Penicka M, Bartunek J, Lang O, Medilek K, Tousek P, Vanderheyden $\mathrm{M}$, et al. Severe left ventricular dyssynchrony is associated with poor prognosis in patients with moderate systolic heart failure undergoing coronary artery bypass grafting. J Am Coll Cardiol 2007;50:1315-23.

19. Lim SP, McArdle BA, Beanlands RS, Hessian RC. Myocardial viability: It is still alive. Semin Nucl Med 2014;44:358-74.

20. Buxton AE, Lee KL, Hafley GE, Pires LA, Fisher JD, Gold MR, et al. Limitations of ejection fraction for prediction of sudden death risk in patients with coronary artery disease: Lessons from the MUSTT study. J Am Coll Cardiol 2007;50:1150-7. 